\title{
SUDDEN HEARING LOSS TREATED WITH HYPERBARIC OXYGEN (HBO) - CASE STUDY
}

\author{
$\underline{\text { Michał Fal }{ }^{1)}}$, Jacek Piechocki ${ }^{2)}$, Monika Morawska-Kochman ${ }^{1)}$, Maria Michałkiewicz ${ }^{1)}$, Tomasz Kręcicki ${ }^{1)}$ \\ ${ }^{1)}$ Clinic of Otolaryngology, Head and Neck Surgery of the Medical University of Wrocław, Poland \\ ${ }^{2)}$ Mazowia Hyperbaric Therapy Centre and Wound Treatment, Warsaw, Poland
}

\begin{abstract}
The article presents a case study of a 32-year-old patient admitted to the Mazowia Hyperbaric Therapy Centre due to a sudden loss of hearing. In an interview the patient reported an abrupt hearing deterioration in his right ear and the feeling of congestion in the left ear with bilateral tinnitus present for over 2 months. The patient was qualified to undergo hyperbaric oxygen therapy (HBO). After 15 treatment sessions in a pressure chamber a noticeable improvement of hearing in his right-ear was noted (proved by pure tone audiometry) and subjective hearing upswing in his left ear.

The bibliography includes the articles and references concerning the time limits of HBO therapy and its effectiveness in the treatment of sudden deafness. Key words: sudden deafness, hyperbaric oxygen, hyperbaric oxygen therapy.
\end{abstract}

ARTICLE INFO

PolHypRes 2015 Vol. 51 Issue 2 pp. 7-10

ISSN: $1734-7009$ elSSN: 2084-0535

DOI: $10.1515 /$ phr-2015-0007

Pages: 4, figures: 2, tables: 0

page www of the periodical: www.phr.net.pl

Publisher

Polish Hyperbaric Medicine and Technology Society

\section{Casuistic article (case study)}

Delivery date: $29.04 .2015 \mathrm{r}$.

Date of approval for print: $12.05 .2015 \mathrm{r}$. 


\section{INTRODUCTION}

The article presents a case study of a patient admitted to the Mazowia Hyperbaric Therapy Centre due to a sudden loss of hearing. Sudden deafness is defined as sensorineural hearing loss of unknown etiology, exceeding $30 \mathrm{~dB}$ and occurring in at least three neighbouring frequencies, which appears abruptly within the period of 3 days. Accompanying symptoms include tinnitus, vertigo, vomiting (20-60\%). There is an absence of cranial symptoms. The incidence reaches 5-20/100 000 cases with probability of occurrence increasing with age. Idiopathic healing occurs in $32-65 \%$ of patients - the majority within the first week [6].

A 32-year-old with an abrupt hearing deterioration in his right ear and the sensation of congestion in the left ear with bilateral tinnitus present for over 2 months. Pure tone audiometry examination confirmed a medium right-side sensorineural hearing loss up to $55 \mathrm{~dB}$ and left-side sensorineural hearing loss limited solely to the frequency of $4000 \mathrm{~Hz} 40 \mathrm{~dB}$.

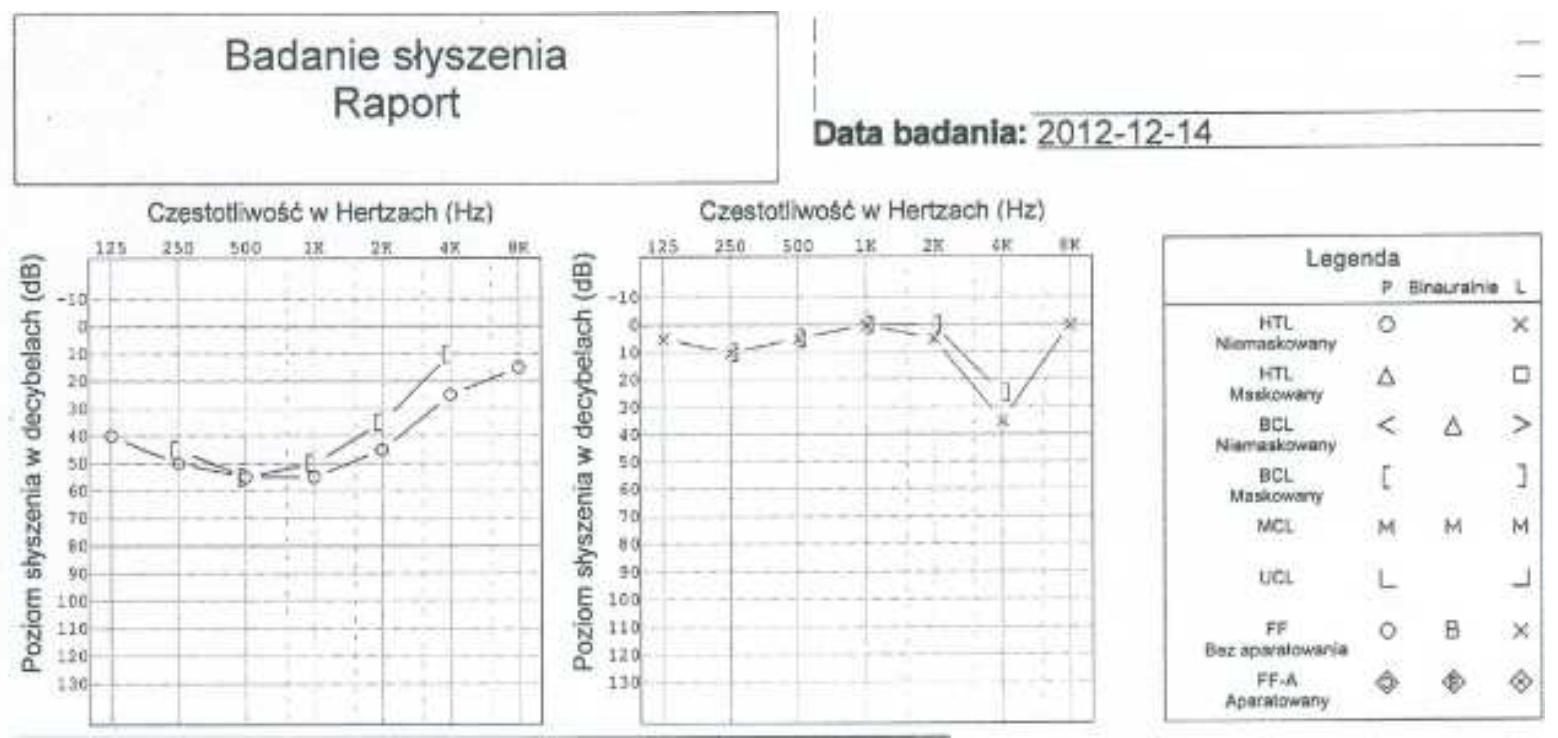

Prior to admittance, pharmacological treatment was introduced at three Otolaryngology Wards to no measurable effect (Vit B1, B6, B12, Adavin, Nootropil, Hydrocortison, Dexaven, Betaserc, Cavinton, Nivalin, biostimulating laser, Xyl-Ca iontophoresis).

Therapy: The patient was qualified to undergo hyperbaric oxygen therapy (HBO) and subjected to 15 sessions. Pharmacological treatment was sustained in the course of hyperbaric therapy (Encoton). Complete recovery in right-ear hearing was observed (confirmed by pure tone audiometry) as well as subjective hearing upswing in the patient's left ear.

\section{iekt.t. 32}
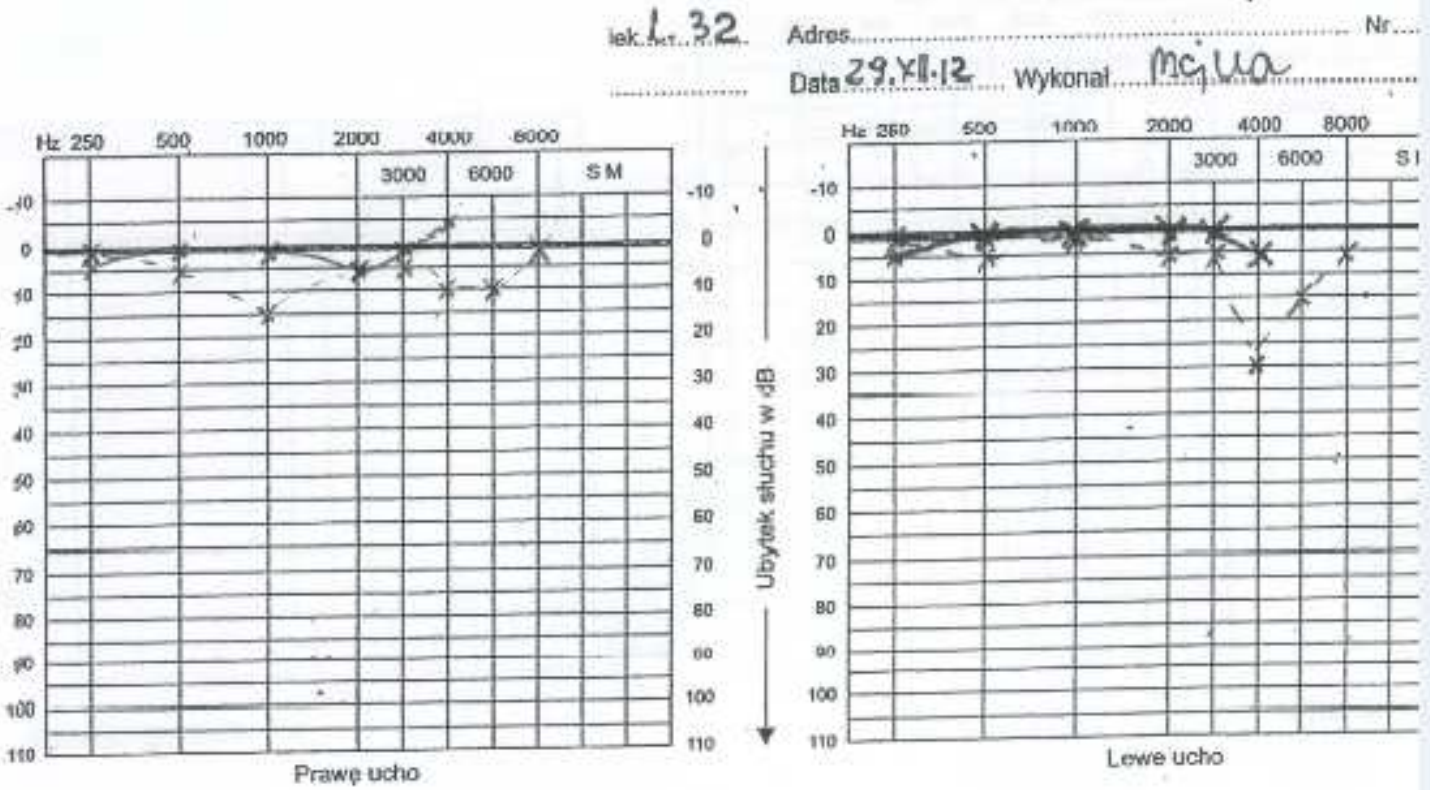


\section{DisCUSSION}

Hyperbaric oxygenation (HBO) is a treatment consisting in the use of oxygen, air or a mixture of oxygen and air at a pressure exceeding 1 atmosphere. Treatment takes place in a hyperbaric chamber where the pressure is 2.5 times higher than atmospheric pressure. HBO causes an increase in the elasticity of erythrocytes and reduces the viscosity of blood, thus improving microcirculation.

One session lasting 1.5 hours consists of several phases. The first phase lasts 10 minutes and includes air compression to the pressure of 2.5 atmospheres (pressure equivalent to the depth of $15 \mathrm{~m}$ under water). This pressure value is maintained for 70 minutes. During this second phase the patient breathes oxygen through a mask, taking 5-minute air breaks. The next phase is the decompression.

In Poland, recommendations for the use of HBO are determined in accordance with the list established by the National Health Fund on the basis of ECHM guidelines [8], deafness being among them.
Hyperbaric therapy is the only known method of increasing p02 in the liquids of the inner ear. When combined with large-dose steroid therapy, hyperbaric therapy gives positive results in $59.7 \%$ of patients [7].

In the available literature and guidelines regarding treatment of abrupt idiopathic deafness, special attention is paid to the time elapsed between the occurrence of symptoms and the commencement of treatment, with emphasis on the necessity of quick therapy implementation.

There is however an absence of guidelines defining the timeframe for the introduction of HBO. The most favourable prognoses concern patients with medium level or deep hearing loss $(>41 \mathrm{~dB})$ for whom HBO was implemented within 14 days of the occurrence of hearing loss. Nonetheless, there have been reports on improvement after 30 days.

The described clinical case points to the validity of undertaking therapy even after a longer period of time (60 days) from the occurrence of sudden idiopathic deafness.

\title{
BIBLIOGRAPHY
}

www.hiperbaria.pl Mazowia Hypebaric Therapy Centre;

J.Piechocki, T.Janus, A. Nielepiec-Jałosińska, J.Sokołowski Introduction to hyperbaric oxygen therapy;

Satar B, Hidir Y, Ytiser S. Effectiveness of Hyperbaric oxygen therapy in idiopatic sudden hearing loss. J Laryngol Otol. 2006; 120: 665-9;

W. Narożny, Z. Sićko, T. Przewoźny, C. Stankiewicz, J. Kot, J. Kuczkowski Usefulness of high doses of glucocorticoids and hyperbaric oxygen therapy in sudden sensorineural hearing loss treatment. Otology \& Neurotology 2004; 25(6): 916-923

5. M. Jadczak, P. Rapiejko, I. Kantor, K. Szczygielski, J.Usowski, J.Piechocki, D.Jurkiewicz The assessment of the performance of treatment of sudden idiopathic hearing loss with the use of hyperbaric oxygen therapy Otolaryngologia Polska 2007 | 61 | 5 | 887-891;

6. Schreiber EB, Agrup Ch, Haskard DO, LuxonLM, Sudden sensorineural hearing loss The Lancet Volume 375, Issue 9721, 3-9 April 2010, Pages 12031211 ;

7. Fujimura T, Suzuki H, Shiomori T, Udaka T,Mori T Hyperbaric oxygen therapy for idiopathic sudden sensorineural hearing loss. Eur. name Arch.Otorhinolaryngology (2007) 264-:861-866;

8. Annex no. 4 to information materials - SOK. In: Annex to ordinance No. 84/2005 of the President of National Health Fund. Detailed information materials on the procedure concerned with conclusion of agreements on the provision of health care services.

\author{
Michał Fal \\ Klinika Otolaryngologii, Chirurgii Głowy i Szyi \\ Uniwersytet Medyczny we Wrocławiu \\ Wybrzeże L. Pasteura 1 \\ 50-367 Wrocław
}

